\title{
Automobile Anti-theft System Design based on GSM
}

\author{
Lili Wan, Tiejun Chen \\ Electrical Engineering College of Zhengzhou University, Henan Zhengzhou, 450001 \\ Wlili2003@sohu.com
}

\begin{abstract}
An automobile remote alarm system based on GSM communication network is introduced. The TC35 GSM module and Microcontroller is combined with the system, vibration sensor and speed sensor are used to achieve short message service (SMS) alarm and dual theft-proof of automobile. The shortcoming of traditional systems including low security and small alarming range is overcome, and the cost of this system is cut down, so it should have good application prospects.
\end{abstract}

\section{Introduction}

Automobile industry and automobile market is in a high speed development state for several years. Automobile's appearance impacts and changes people's life, it's becoming the progressive symbol of modern society. However, as the sharp rise of the automobile quantity, vehicle thief case is increasing. Not only anti-theft system' demand is increasing, but also the performance requirement is higher and higher. At present, in the leading market of the automobile anti-theft production is CMOS chip production. GPS positioning system is also used, or combine the CMOS chip and GPS positioning system ${ }^{[1-3]}$. However, chip anti-theft production is with a low security and small alarm scope, which can't be found after lost yet. Although GPS can used to retrieve automobile, what make user flinch is the high cost. Use GSM network can work out an anti-theft system with low cost, large alarm scope, strong Anti-jamming capability, which even be able to verify approximate positioning in a certain scope ${ }^{[4]}$.

The automobile theft-proof system based on GSM communication network designed in this paper be able to accomplish short message services(SMS) like homogeneous anti-theft system, moreover, speed sensor and vibrating sensor are combined to realize dual theft-proof of automobile. The fortified vehicular security is advanced greatly, and the cost of the automobile's orient is reduced.

\section{System construction}

The system mostly be composed of TC35 GSM network module, vibrating sensor, speed sensor, A / D convertor, micro-processing controller and user handset.

The working principle of the whole system is: The car is in a state of maximum security when the owner leaves. The sensors begin to work, and send the signal which is converted by the A / D converter to the micro-processing chip in time. The first anti-theft: The vibration sensors are used to detect signals. When the doors are open illegally or car crash is severe, sound and light alarm, cut off oil, send message to notify the owner at the same time. If it don't receive an order to cancel the warning in two minutes, dial the owner's phone through which can monitor the situation in the car; the second tier of anti-theft: Speed detection sensors are used to detect signals. If the first theft-proof is fail, car was started by the thief, once the speed is higher than $15 \mathrm{~m} / \mathrm{s}$, the oil is cut off again, sound and light alarm, dial the owner's phone. If 
the two anti-thefts are failed, on-board communications device signals can be base-station searched through GSM mobile communications network, which can provide clues for retrieving vehicle.

Block diagram of system construction is shown in Figure 1.

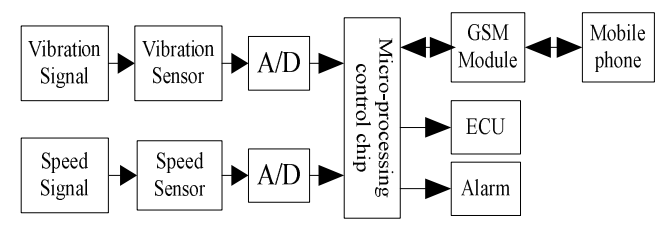

Figure1. Block diagram of system construction

\section{TC35 GSM module}

TC35 module is selected. It is a new wireless module produced by Siemens. It can realize data and voice transmission quickly and safely, such as short message services SMS and fax. It can work in GSM $900 \mathrm{kHz}$ and $1800 \mathrm{kHz}$ two bands. RS232 data port tally with ETSI standard GSM0707 and GSM0705. It's easy to be upgraded to GPRS module. The module which set RF Circuits and base-band together provides users with the standard AT command interface can make fast, reliable, secure transmission of data, voice, fax and SMS. It's easy for users to develop and design. TC35 module is composed mainly by six parts: GSM base band processor, GSM radio frequency modules, power modules (ASIC), flash memory, ZIF connector, antenna interface. The function block diagram is shown in Figure 2. As the core of TC35, base band processor with the responsibility of disposing voice and data signal in the terminal of GSM, and covers all of the analog and digital functions in cellular radio equipment. It supports FR, HR and EFR voice channel coding with no additional hardware circuitry premise. Its detailed instruction set please refer to literature [9].

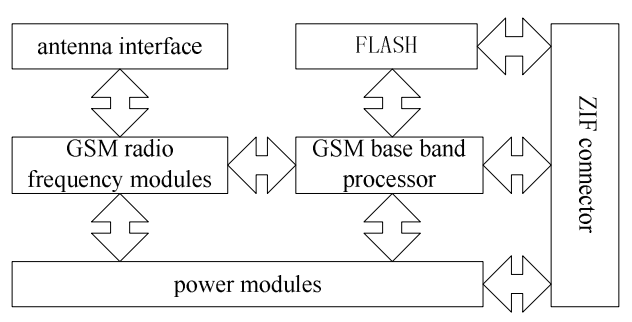

Figure2. Functional block diagram of TC35

\section{Hardware Design}

\subsection{Hardware Overall Design}

The hardware is mainly composed of five parts: controller, TC35 GSM module, vibration sensor, speed sensor and A / D converter.

As the main control unit in the system, Single chip plays a central role. AT89C52 of ATMEL Corporation which is a low-power, high-performance single-chip with $8 \mathrm{~K}$ bytes internal Flash Memory is selected. Advanced high-density non-volatile memory technology of ATMEL is used in the chip. It's fully compatible with instruction set and output pins of 51 series. There are 256 bytes RAM, 32 programmable I / O Line, 3 16-bit timing counters, 6 Two-stage interrupt sources, and a full-duplex serial port interiorly.

Vibration sensors are put on four doors, font cover and back six places. SC0063 displacement vibration sensor is selected. It isn't restricted by the direction of librations, and its perceived sensitivity are the same whatever at the upper and lower, left and right, front and rear. The sensors are between the IC trigger-end and power of general alarm sensors. It will be triggered alarms by a degree of vibration. The circuit is shown in Figure 3.

Photoelectric speed sensor configuration is used in the speed sensor, which is constituted of light-emitting diodes, Phototransistor and light shade board. Its working principle is shown in Figure 4. When the light shading board can not Trip the speed of light, light of LED reach the 
Phototransistor, current pass the collector of Phototransistor, then the tube turn on, transistor VT1 also turn on, so the Si terminal output $5 \mathrm{~V}$ voltage. Pulse frequency is decided by the speed.

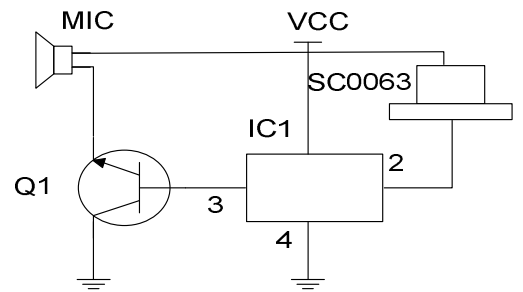

Figure3. Warning circuit chart of SC0063

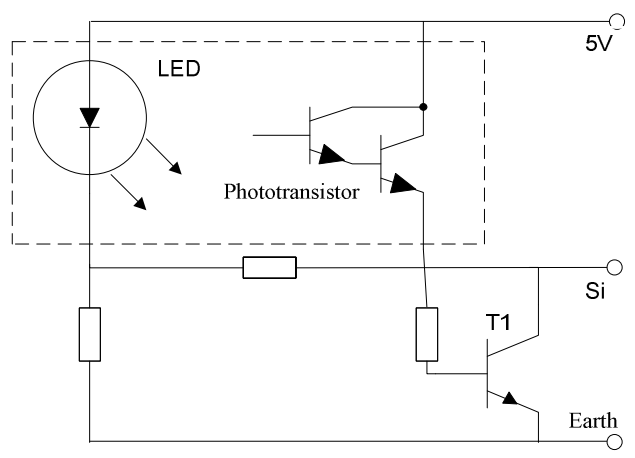

Figure4. Circuit diagram of photoelectrical vehicle speed sensor

\subsection{Interface design}

4.2.1. Interface circuit design of GSM and AT89C52 TC35's start, reset and shut down is controlled by $\mathrm{P} 1.0$ and $\mathrm{P} 1.1$ of the single-chip. Data communications with TC35 through the serial port, by communication rate of $9600 \mathrm{Kbps}$, using 8-bit asynchronous communication: a start bit, 8 data bits, a stop bit. The essential part of the interface between single-chip and TC35 is shown in figure 5. It should be noted that:

1) TTL logic-positive of TC35 module's input and output is not $+5 \mathrm{~V}$, but $+2.9 \mathrm{~V}$. Therefore, input level must have a logic conversion by adding pull-up resistor to the output of the open-collector buffer 7407 .

2) After power the system, in order to make TC35 working, must plus a low pulse which is more than $100 \mathrm{~ms}$ delay to IGT. The duration of level decreased is no more than $1 \mathrm{~ms}$. After the start, IGT should remain high (3.3V). Supply voltage of TC35 can not be lower than $3.3 \mathrm{~V}$ when IGT drive or TC35 can not be activated.

3) CCIN pins of ZIF connector blocks are used to detect whether the SIM card is inserted. If connect right, CCIN pin output high, or low.

\subsubsection{Interface design of AT89C52 and A/D} converter Serial A / D TLC0831 is 8-bit approaches the comparison voltage gradually serial A / D converter, which is 8-pin package. It support single-channel analog input, A / D results serial digital output, without addressing. There is an internal sampled data comparator, which after the input analog signal Differential comparison convert to digital signal. Digital signal is distributed to serial output by Standard shift register. Analog voltage can be differential input to suppress common-mode signal and reduce or eliminate the offset error of conversion. The interface circuit of AT89C52 and A/D is shown in Figure 6:

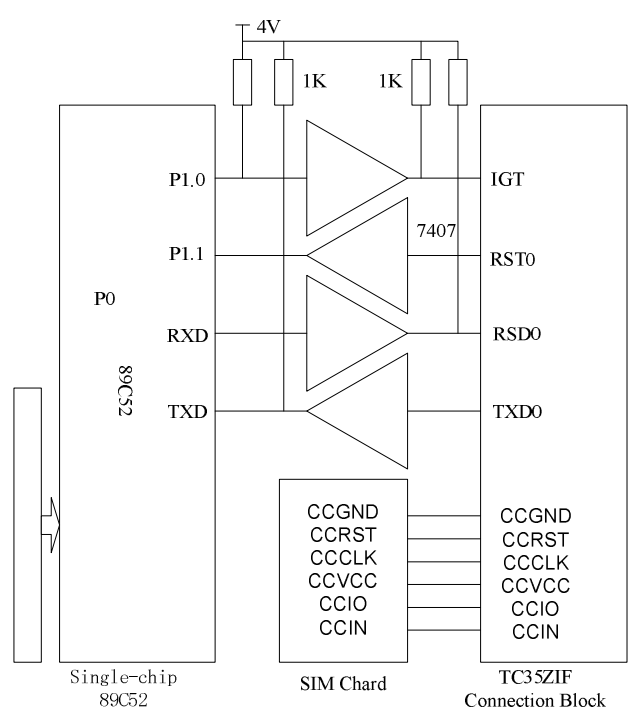

Fig.5 Interface circuit of GSM and

AT89 


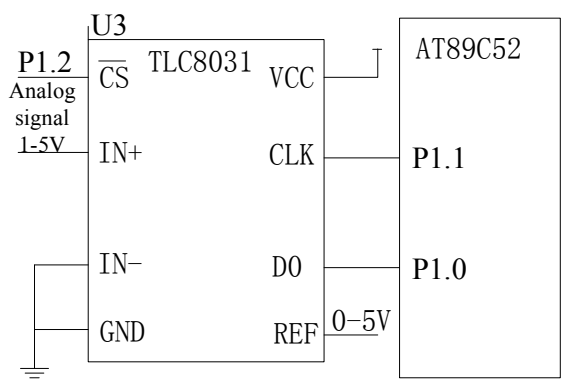

Fig.6 Interface circuit of TLC8031 and AT89C52

\section{Software overall design}

C51 is selected because the programming is high efficiency, accuracy and stability. software design flow chart is shown in Figure 7.

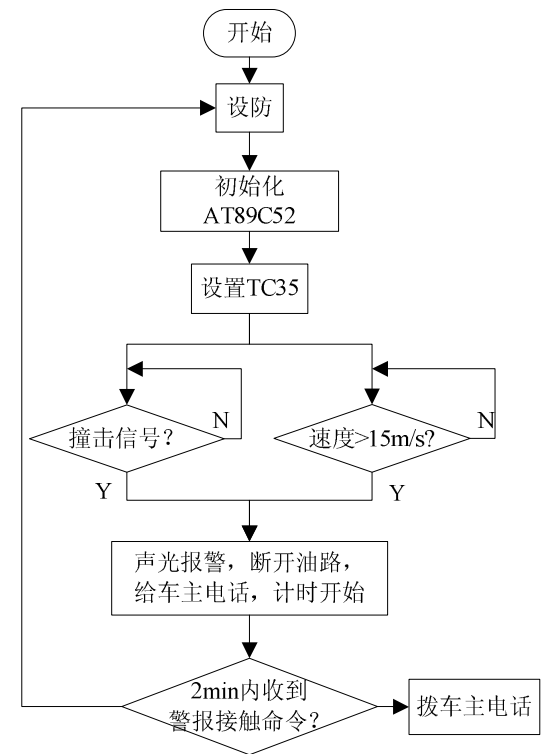

Fig.7 Flow chart of software overall design

\section{Conclusion}

A new GSM-based vehicle anti-theft system is introduced in this paper. Speed sensors and vibration sensors are used to achieve dual theft-proof of automobile. Owner can receive the alarm message quickly and accurately, also can monitor the car by phone if necessary. The low-cost system has achievability and good usability. With the development of information technology, GSM networks will be perfect and the system will have better prospects.

\section{References}

[1] Cui Gengshen, and Huang Tinghui. A study on the auto guard against theft sestem of network based on S3C44B0X[J]. Microcomputer Information, Guang Xi, 2006, 22(11-2): 146-148.

[2] Wei Zhenlin, and Wang Xifu. Design of Real-time Monitor System for Electric Vehicles Based on GPS[J]. Journal of China Safety Science, Bei Jing, 2006, 16(4): 136-140.

[3] Zhai Guorui, and Dai Shenghua. Design of auto guard against theft system based on GPRS and GPS[J]. Microcontrollers and Embedded Systems, Bei Jing, 2007, 8: 39-41.

[4] Fan Zhenfang, Peng Aihua, and Zhou Jian, etc. Design of auto guard against theft and alarm system based on GSM network[J]. Application of Electronic Technique, Chang Sha, 2006, 3: 14-16.

[5] Song Fuchang. Identification and detection diagram of automobile sensor[M]. Beijing: Electronic Industry Press. 2003.

[6] ZHENG Youzheng. Single chip principle and application[M]. Sichuan: Sichuan University Press, 2003.

[7] Zhao Futu. Sensor integrated circuit manual. Beijing: Chemical Industry Press. 2003.

[8] Xavier Lagrange, Philippe Godlewski, Sami Tabbane. GSM network and GPRS[M]. Beijing: Electronic Industry Press. 2002.

[9] Siemens Inc. Siemens Cellular Engines AT Command Set [EB/OL]. 2005-01- 21. 